\title{
Avaliação das habilidades Cognitivas, da COMUniCaÇão E NEUROMOTORAS DE CRIANÇAS COM RISCO DE ALTERAÇÕES DO DESENVOLVIMENTO ${ }^{1}$
}

ASSESSMENT OF THE COGNITIVE, COMMUNICATION AND NEUROMOTOR SKILLS

OF CHILDREN AT RISK FOR DEVELOPMENTAL ALTERATIONS

Andréia da Cunha Tavares AMARAL ${ }^{2}$ Maria de Lourdes Merighi TABAQUIM ${ }^{3}$ Dionísia Aparecida Cusin LAMÔNICA ${ }^{4}$

\begin{abstract}
RESUMO: o objetivo deste estudo foi avaliar habilidades neuropsicomotoras e funcionais de crianças que apresentaram, no primeiro ano de vida, história clínica de risco para alterações do desenvolvimento, quanto às áreas motora, cognitiva, lingüística e psicossocial. Foram avaliadas 60 crianças, classificadas em dois grupos: o Grupo Experimental (GI), composto por 30 crianças com idade cronológica variando de zero a dois anos e onze meses, ambos os sexos, tendo história de intercorrências peri, pré e pós-natal e/ou atraso neuropsicomotor; o Grupo Controle (GII), formado por 30 crianças sem história de alterações do desenvolvimento, pareadas quanto ao sexo e idade. Os resultados indicaram que, na comparação entre grupos, (GI e GII), GI apresentou diferença estatisticamente significante nas áreas motora, de linguagem e cognitiva. Na análise da comparação entre áreas, as de maior prejuízo foram a da linguagem e motora, seguido da cognitiva. A área psicossocial foi a menos comprometida e na comparação entre grupos os escores não foram estatisticamente significantes. Isto demonstra a influência dos fatores de risco no processo de desenvolvimento e a importância do acompanhamento precoce às crianças que apresentam histórico de intercorrências.
\end{abstract}

PALAVRAS-CHAVE: linguagem, desenvolvimento psicomotor, socialização, educação especial.

ABSTRACT: the objective of this study was to assess the functional and neuropsychomotor skills of children who presented a clinical history of a risk for developmental alterations during their first year of life in the motor, cognitive, linguistic and psychosocial areas. Sixty children were assessed and classified into two groups: the Experimental Group (GI), made up of 30 children ranging from zero to two years and eleven months old, of both sexes, with a history of peri-, pre- and postnatal intercurrences and/or neuropsychomotor delay; the Control Group (GII), made up of 30 children without any history of developmental alterations, and matched according to sex and age. When the two groups (GI and GII) were compared, the results revealed a statistically significant difference in GI in the motor, language and cognitive areas. In the comparative analysis between the different areas, those that revealed the biggest detriment were the language and motor skills, followed by the cognitive skills. The psychosocial area was the least compromised and in the comparison between the two groups the scores were not statistically significant. This shows the influence of risk factors in the developmental process and the importance of an early follow-up with those children that reveal a history of intercurrences.

KEYWORDS: language, psychomotor development, socialization, special education.

\footnotetext{
1 Apoio PIBIC/CNPq.

${ }^{2}$ Discente do Curso de Fonoaudiologia da Universidade do Sagrado Coração. E-mail: andreiamaral@igzipmail

${ }^{3}$ Professora Doutora do Curso de Psicologia da Universidade do Sagrado Coração de Bauru E-mail: ptabaqui@uol.com.br

${ }^{4}$ Professora Doutora do Departamento de Fonoaudiologia da Faculdade de Odontologia da Universidade de São Paulo - FOB/USP-Bauru. E-mail: $\underline{\text { dionelam@uol.com.br }}$
} 


\section{INTRODUÇÃO}

O termo alto risco geralmente, está relacionado com variáveis biológicas, ambientais e/ou psicológicas, em que o alto risco está direcionado para a possibilidade de alterações do desenvolvimento (SUSSMAN et al. 1996).

Diferentes concepções biológicas inferem que alterações iniciais na formação e maturação biológicas do bebê podem trazer interferência no processo normal de evolução física, motora, auditiva e psicológica da criança, influenciando no processo de aquisição e desenvolvimento de maneira global (GERBER, 1996).

A melhoria das condições de atendimento à gestantes e o avanço de tecnologias médicas e melhoria de atendimento na saúde pública, têm favorecido a sobrevida de crianças que passaram por eventos de risco e, desta forma, têm se observado aumento da incidência de alterações de desenvolvimento em crianças que apresentaram histórico de injúrias durante gestação e/ ou parto (BERGER, 2003).

A população de risco depende de cuidados especiais desde o nascimento, uma vez que estes recém-nascidos estão mais participantes a uma série de doenças que podem ocasionar alterações em seu desenvolvimento. (DIAMENT; CYPEL, 1996).

A maioria dos sobreviventes, devido às complicações pré e peri-natais desenvolve, mesmo que tardiamente, senão precoce, distúrbios múltiplos na primeira infância ou idade escolar, incluindo dificuldades de aprendizagem, da integração viso motora, de linguagem e distúrbios de comportamento (FONSECA, 1996).

Segundo estudos (PIOVESANA, 1998; TABAQUIM, 2002; MILLER, CLARK, 2002), dentre os fatores de risco a prematuridade e o baixo peso são fatores relevantes e crianças com este histórico deveriam ser acompanhadas no sentido de prevenir alterações de desenvolvimento. Para Piovesana (2001), o foco de atenção é a substância branca, pois a condição hipóxico-isquêmica prediz o risco de anormalidades no sistema nervoso. No prematuro, durante o período pós-parto há fatores de risco importantes como a baixa idade gestacional pneumotórax, apnéia sangüínea. Entretanto, no sistema nervoso do prematuro, embora mais vulnerável, também existe a ação de fenômenos plástico-regenerativos que promovem a reorganização neurológica, importante nos processos de adaptação. Atualmente, a maioria de trabalhos procura estudar os fatores de risco por meio dos aspectos clínicos e neuropatológicos de acordo com o momento, real ou suposto, do insulto ou da intercorrência.

O desenvolvimento motor, quando ocorre com atraso, costuma seguir uma seqüência própria, desorganizada, dependente da etiologia específica que levou a este atraso, trazendo conseqüências importantes nas interações que a criança faz no seu ambiente, podendo promover alterações secundárias com influência imediata no desempenho de habilidade de vida diária (MILLER; CLARK, 2002).

O desenvolvimento motor é fundamental, considerando que a criança desenvolve sua linguagem no intercâmbio com o ambiente, pela exploração ativa 
por meio da manipulação dos objetos, da repetição das ações, pelo domínio do próprio corpo e controle do esquema corporal e pelas relações que estabelece no ambiente (LAMÔNICA, 2004).

Segundo Piaget (ATKINSON et al, 2002) a criança no primeiro ano de vida apresenta evolução significativa do desenvolvimento nos aspectos psicossociais, quando recebe grande influência da mãe e do ambiente, e principalmente favorece a evolução de aquisições cognitivas movidas pelas ações intencionais promovendo desta forma a aprendizagem.

Durante muitos anos o desenvolvimento motor foi descrito como uma tomada de consciência progressiva, pela criança, de seu sistema muscular, na mesma medida da inibição dos atos reflexos, da progressão das habilidades e da repetição das experiências motoras (COHEN, 2001).

As primeiras aquisições motoras do bebê foram descritas como partindo de movimentos simples para movimentos cada vez mais complexos (BACKLIN, 1992). Respeitando as variações individuais relativas ao desenvolvimento a expectativa de que aos 3 meses a criança já tenha adquirido o controle cervical, aos 6 meses sente-se sem apoio e aos doze meses consiga marcha independente. Esta fase, é de grande importância pois a criança pode explorar, com independência, seu ambiente. Isso coincide grande evolução da maturação do sistema nervoso central, salto no desenvolvimento cognitivo e afetivo e comunicativo, melhora da qualidade das informações exteroceptivas e proprioceptivas e riqueza das experiências motoras em função do meio onde elas se desenvolvem. O conjunto desses parâmetros interage no contexto cultural e social onde vive a criança devendo ser levado em conta na presença de atraso do desenvolvimento.

Cipriani e Bruni (1986) investigaram a relação entre as habilidades receptivas de crianças com lesões neurológicas e suas possibilidades de realização neuropsicomotoras, Os resultados apontaram que as crianças sem atraso do desenvolvimento neuropsicomotor apresentaram escores muito superiores nas habilidades receptivas lingüísticas.

A criança nasce equipada com a habilidade para produzir numerosos padrões interativos e sensório-motores, pré-programados para atingir a vinculação essencial à sua sobrevivência e segurança gravitacional, isto é, controle sobre o corpo, motricidade e simultaneamente sobre suas emoções. Assim, os mecanismos cognitivos são baseados na atividade motora, que constituem os primeiros substratos da cognição. Para Gerber (1996), a ação gera e elicita a cognição, através das práxis, que tem por base uma planificação mental que regula, controle, integra, elabora e executa a intenção da criança.

Pheifer (1997) elaborou um instrumento para avaliar habilidades cognitivas de quatro grupos de crianças de ambos os sexos de trinta e seis meses de idade. OGrupo 1 foi composto por crianças sem histórico de risco e sem alteração do desenvolvimento motor. O Grupo 2 por hemiplégicos; o Grupo3 por diplégicos 
e o Grupo 4 por quadriplégicos. As habilidades avaliadas foram: Associação de vocabulário, memória, esquema corporal, discriminação tátil, orientação espacial e temporal. Foi observado que na prova de associação de vocabulário, esquema corporal, discriminação e orientação temporal houve decréscimo da porcentagem de acerto em função do aumento do comprometimento motor. Na prova de vocabulário, não foi encontrada diferença estatisticamente significante nos percentuais de acerto nos quatro grupos, entretanto, o grupo de melhor média foi o grupo 1 seguido do grupo 2, 3 e 4 . O autor afirmou que o vocabulário não pareceu estar sendo influenciado diretamente pelo transtorno motor mas sim pelo grau de interação da criança com outras pessoas. Na prova de memória houve diferença estatisticamente significante entre os grupos 3 e 4, que apresentaram resultados muito abaixo do esperado, o grupo 1 obteve o melhor desempenho. O autor concluiu que nos casos de problemas motores poderia haver imaturidade no desenvolvimento em função da pouca exploração ambiental, norteada pelo atraso do desenvolvimento neuropsicomotor.

Existe o consenso de que crianças acompanhadas, com procedimentos de estimulação, quando identificados precocemente sinais de anormalidade e/ou desvios, demonstram melhoria na condição de respostas e rearranjos na plasticidade cerebral, capazes de minimizar impacto no desenvolvimento estrutural e funcional, proporcionando melhora da qualidade de vida.

Desta forma, neste período, o diagnóstico precoce associado a procedimentos de intervenção interdisciplinar, é fundamental para a melhoria dão desenvolvimento físico, mental, percepto-sensorial e afetivo da criança de risco.

Permeando as hipóteses de que, a exposição as intercorrências que possam levar a alterações do desenvolvimento, condição esta significativa nas fases iniciais do desenvolvimento quando o cérebro está imaturo, torna esta criança vulnerável a eventos interferentes nos processos de aquisição de habilidades, este estudo foi delineado.

Diante o exposto, este estudo tem como objetivo avaliar habilidades neuropsicomotoras e funcionais de crianças que apresentaram, no primeiro ano de vida, história clínica de risco para alterações do desenvolvimento, quanto às áreas motora, cognitiva,lingüística e psicossocial.

\section{Material e MÉtodo}

O projeto foi encaminhado ao Comitê de Ética em Seres Humanos da Universidade do Sagrado Coração, sendo aprovado (Protocolo número 045/2003). Ressalta-se que foram respeitados todos os princípios éticos, conforme versa a Resolução 196/96 do CONEP. Os pais foram convidados a participarem com seus filhos como voluntários e, esclarecidos os objetivos e procedimentos, assinaram o termo de consentimento livre e esclarecido pós-informado. 
Fizeram parte deste estudo, 60 crianças que compuseram dois grupos : Grupo Experimental - GI, com 30 crianças, de ambos os sexos, tendo como critério de elegibilidade história de intercorrências peri, pré e pós-natal e atraso neuropsicomotor, com idade cronológica de zero a dois anos e onze meses de idade e o Grupo Controle - GII, formado por 30 crianças, sem histórico de intercorrências peri, pré e pós-natais e atraso do desenvolvimento, pareado quanto à idade, sexo e nível sócio-cultural.

Para atingir os critérios de elegibilidade, os candidatos ao GI passaram pelo Setor de Neurologia da Clínica de Educação para a Saúde, para exame neurológico. Aos candidatos ao GII, foi realizada uma sessão de anamnese, na qual foi constatada a ausência de risco e alteração do desenvolvimento.

Os encaminhamentos dos candidatos do GI foram realizados pela Clínica de Psicologia Aplicada e Fonoaudiologia, Clínica de Educação para a Saúde e Clínica de Fisioterapia da Universidade do Sagrado Coração - USC/Bauru. Para a formação do GII foram contatadas três creches municipais, na busca pelo pareamento em idade, sexo e nível sócio cultural. Adotou-se como critério aceitar a diferença até dois meses de idade no pareamento das crianças.

As avaliações foram conduzidas na Clínica de Psicologia Aplicada e Fonoaudiologia da Universidade do Sagrado Coração USC/Bauru, com atendimento individual e horário pré-determinado.

O instrumento utilizado na avaliação constou do Protocolo integrado, proposto por Saes (2003).

O protocolo de desenvolvimento é composto por provas distribuídas em oito trimestres com itens de avaliação referente a aspectos do desenvolvimento que foram classificados em quatro módulos: motor $(\mathrm{M})$, cognitivo $(\mathrm{C})$, lingüístico (L) e comportamentos psicossociais $(\mathrm{P})$. As provas são distribuídas por trimestre, $1^{\circ}$ trimestre: 32 provas; $2^{\circ}$ trimestre: 22 provas; $3^{\circ}$ trimestre: 20 provas; $4^{\circ}$ trimestre: 21 provas; $5^{\circ}$ trimestre: 20 provas; $6^{\circ}$ trimestre: 20 provas; $7^{\circ}$ trimestre:13 provas; $8^{\circ}$ trimestre: 7 provas; $9^{\circ}$ e $10^{\circ}$ trimestre: 7 provas. Para as crianças com idade entre 25 e 35 meses, o procedimento adotado foi classificá-las de acordo com a pontuação do último trimestre do instrumento empregado (que previa até 24 meses). Este critério justificou-se pelos comportamentos e aquisições das mesmas encontraremse num período de maturação neurobiológica significativamente próxima à fase sensório-motora, como os demais participantes do estudo.

Desta forma, cada prova atendeu a mensuração de cada uma das áreas investigadas, nas diferentes faixas etárias e do desenvolvimento.

A Tabela 1 apresenta a quantidade de tarefas por área e por fase do desenvolvimento, proposta do Protocolo de Saes (2003). 
Tabela 1 - Representação numérica da quantidade de provas por fase do desenvolvimento trimestral e área avaliada.

\begin{tabular}{ccccc}
\hline Trimestre & Motor & Cognição & Linguagem & Psicossocial \\
\hline $1^{\mathbf{o}}$ & 11 & 7 & 6 & 8 \\
$2^{\mathbf{o}}$ & 9 & 4 & 5 & 4 \\
$3^{\mathbf{o}}$ & 7 & 3 & 5 & 5 \\
$4^{\mathbf{0}}$ & 7 & 4 & 4 & 6 \\
$5^{\mathbf{0}}$ & 13 & 3 & 2 & 2 \\
$6^{\mathbf{0}}$ & 9 & 4 & 4 & 3 \\
$7^{\mathbf{0}}$ & 8 & 2 & 1 & 2 \\
$8^{\mathbf{0}}$ & 1 & 1 & 4 & 1 \\
$9^{\mathbf{0}}$ & 1 & 1 & 4 & 1 \\
$10^{\mathbf{0}}$ & 1 & 1 & 4 & 1 \\
\hline
\end{tabular}

O protocolo de avaliação das habilidades motoras, cognitivas, da comunicação e do comportamento psicossocial foi aplicado diretamente à criança e complementado com as informações prestadas pelas mães. As habilidades demonstradas pela criança foram pareadas aos critérios do protocolo, estabelecendo a equivalência aos meses do desenvolvimento e idade. As pontuações obtidas foram transformadas percentualmente, efetuadas às média aritméticas e submetidas ao tratamento estatístico.

Além de apresentar os dados estatísticos em termos percentuais optouse pela análise estatística, utilizando-se testes não paramétricos. Os testes Kruskal Wallis e Teste de Milller foram aplicados, fixando o nível de significância em 0,05\% ou $5 \%$. O objetivo do uso destes testes foi comparar o desempenho entre grupos e entre as áreas.

\section{Resultados}

Caracterização da população estudada

O Quadro 1 apresenta a caracterização dos grupos experimental (GI) e controle (GII), quanto ao sexo e idade. 
Quadro 1 - Caracterização dos grupos (GI e GII), quanto ao sexo (M= masculino e $\mathrm{F}=$ Feminino $)$ e idade $(\mathrm{m}=$ meses $)$.

\begin{tabular}{|c|c|c|c|c|c||}
\hline \multicolumn{3}{|c|}{ Grupo Experimental - GI } & \multicolumn{3}{c|}{ Grupo Controle - GII } \\
\hline Participante & Sexo & Idade & Participante & Sexo & Idade \\
\hline 01 & M & $18 \mathrm{~m}$ & 01 & M & $20 \mathrm{~m}$ \\
\hline 02 & M & $19 \mathrm{~m}$ & 02 & M & $18 \mathrm{~m}$ \\
\hline 03 & M & $14 \mathrm{~m}$ & 03 & M & $16 \mathrm{~m}$ \\
\hline 04 & M & $26 \mathrm{~m}$ & 04 & M & $27 \mathrm{~m}$ \\
\hline 05 & F & $20 \mathrm{~m}$ & 05 & F & $20 \mathrm{~m}$ \\
\hline 06 & F & $26 \mathrm{~m}$ & 06 & F & $28 \mathrm{~m}$ \\
\hline 07 & M & $22 \mathrm{~m}$ & 07 & M & $24 \mathrm{~m}$ \\
\hline 08 & M & $24 \mathrm{~m}$ & 08 & M & $24 \mathrm{~m}$ \\
\hline 09 & F & $18 \mathrm{~m}$ & 09 & F & $18 \mathrm{~m}$ \\
\hline 10 & F & $15 \mathrm{~m}$ & 10 & F & $15 \mathrm{~m}$ \\
\hline 11 & F & $18 \mathrm{~m}$ & 11 & F & $18 \mathrm{~m}$ \\
\hline 12 & F & $5 \mathrm{~m}$ & 12 & F & $05 \mathrm{~m}$ \\
\hline 13 & M & $13 \mathrm{~m}$ & 13 & M & $13 \mathrm{~m}$ \\
\hline 14 & M & $26 \mathrm{~m}$ & 14 & M & $26 \mathrm{~m}$ \\
\hline 15 & F & $21 \mathrm{~m}$ & 15 & F & $22 \mathrm{~m}$ \\
\hline 16 & M & $34 \mathrm{~m}$ & 16 & M & $34 \mathrm{~m}$ \\
\hline 17 & M & $25 \mathrm{~m}$ & 17 & M & $24 \mathrm{~m}$ \\
\hline 18 & F & $28 \mathrm{~m}$ & 18 & F & $27 \mathrm{~m}$ \\
\hline 19 & M & $16 \mathrm{~m}$ & 19 & M & $16 \mathrm{~m}$ \\
\hline 20 & M & $16 \mathrm{~m}$ & 20 & M & $16 \mathrm{~m}$ \\
\hline 21 & M & $16 \mathrm{~m}$ & 21 & M & $14 \mathrm{~m}$ \\
\hline 22 & F & $13 \mathrm{~m}$ & 22 & F & $13 \mathrm{~m}$ \\
\hline 23 & M & $22 \mathrm{~m}$ & 23 & M & $22 \mathrm{~m}$ \\
\hline 24 & M & $25 \mathrm{~m}$ & 24 & M & $25 \mathrm{~m}$ \\
\hline 25 & F & $27 \mathrm{~m}$ & 25 & F & $27 \mathrm{~m}$ \\
\hline 26 & F & $16 \mathrm{~m}$ & 26 & F & $16 \mathrm{~m}$ \\
\hline 27 & F & $16 \mathrm{~m}$ & 27 & F & $16 \mathrm{~m}$ \\
\hline 28 & F & $16 \mathrm{~m}$ & 28 & F & $18 \mathrm{~m}$ \\
\hline 29 & F & $16 \mathrm{~m}$ & 29 & F & $16 \mathrm{~m}$ \\
\hline 30 & M & $09 \mathrm{~m}$ & 30 & M & $09 \mathrm{~m}$ \\
\hline
\end{tabular}

O Quadro 2 apresenta a representação etiológica descrita pela mãe no momento da anamnese e no prontuário da avaliação neurológica, quanto às condições pré, peri e pós-gestacionais. 
Quadro 2 - Representação etiológica do GI com a descrição de intercorrências pré, peri e pós-natal. (PT: pré termo; AT: a termo).

\begin{tabular}{|c|c|}
\hline Sj & Histórico \\
\hline 1 & PT, pré-eclâmpsia, baixo peso, insuficiência respiratória, Icterícia, sonda oro e nasográstrica. \\
\hline 2 & PT, baixo peso (1500kg), (hipoxemia), cianose, incubadora. \\
\hline 3 & AT, não chorou, uso de fórceps, anóxia, incubadora, UTI 40 dias, convulsões. \\
\hline 4 & AT, convulsivo, cardiopatia congênita. \\
\hline 5 & AT, 3600kg, macrossomia, diabetes materno, casal consangüíneo. \\
\hline 6 & AT, hidrocefalia, ptose palpebral \\
\hline 7 & AT, ausência de choro, apgar baixo \\
\hline 8 & PT, 5 abortos anteriores, infecção hospitalar, icterícia, cirurgia de hérnia. \\
\hline 9 & PT, reflexos alterados, insuficiência cárdio-pulmonar, pneumonia, sonda nasogástrica. \\
\hline 10 & Diabete materna, macrossomia, anóxia, icterícia, convulsões. \\
\hline 11 & PT, baixo peso, incubadora. \\
\hline 12 & PT, incubadora com respiração artificial. \\
\hline 13 & PT, ausência do choro. \\
\hline 14 & PT, inércia uterina, inativo, sofrimento fetal, convulsão neonatal, incubadora, icterícia, anóxia. \\
\hline 15 & PT, gemelar, descolamento de placenta, incubadora, respiração artif., pneumotórax, apnéia, hidrocefalia. \\
\hline 16 & PT, mioma uterino, pneumonia, incubadora, deficiência visual, hipóxia, convulsões. \\
\hline 17 & AT, convulsão, intervenção cirúrgica de abscesso cervical aos 4 meses. \\
\hline 18 & PT, ausência de chorou, incubadora, sonda nasogástrica. \\
\hline 19 & PT, gestação trigemelar, baixo peso, incubadora. \\
\hline 20 & PT, gestação trigemelar, baixo peso, incubadora. \\
\hline 21 & PT, gestação trigemelar, baixo peso, incubadora. \\
\hline 22 & AT, liquido amniótico aspirado, incubadora, parada cardíaca, convulsões. \\
\hline 23 & PT, 3 abortos anteriores, ausência de chorou, anóxia, cianose, incubadora. \\
\hline 24 & $\begin{array}{l}\text { PT, diabetes materna, estado comatoso mãe/bebê, hemodiálise, choro frágil, anóxia, insuficiência cárdio-respiratória, } \\
\text { convulsões. }\end{array}$ \\
\hline 25 & PT, choro frágil, inércia e hipotonia, incubadora. \\
\hline 26 & PT, pré-eclâmpsia, gestação gemelar, anóxia, baixo peso, incubadora, sonda nasogástrica. \\
\hline 27 & PT, pré - eclampsia materna, gestação gemelar, baixo peso, incubadora. \\
\hline 28 & PT, deslocamento de placenta, gestação gemelar, incubadora. \\
\hline 29 & PT, pré - eclampsia materna, ausência de chorou, baixo peso, incubadora. \\
\hline 30 & PT, icterícia, incubadora, baixo peso, anóxia. \\
\hline
\end{tabular}

Constatou-se que 74\% tiveram nascimento prematuro (PT), 60\% histórico de hipoxemia, $74 \%$ baixo peso, 36\% crises convulsivas, $13 \%$ gestação gemelar e trigemelar e cardiopatia congênita $6 \%$ hidrocefalia, icterícia, diabetes, pré-eclâmpsia maternos; além de outros fatores instrumentais e teratogênicos. 
Dados da aplicação do protocolo quanto ao desempenho dos participantes

A Tabela 2 e 3 demonstra os resultados obtidos pelos grupos GI e GII, descrevendo os escores esperados (ES), atingidos (At), para as áreas motora, cognição, linguagem, psicossocial e a porcentagem de acertos.

Tabela 2 - Representação numérica, por área, e média percentual dos desempenhos do GI, esperados (Es) e atingidos (At).

\begin{tabular}{|c|c|c|c|c|c|c|c|c|c|c|}
\hline \multirow[t]{2}{*}{ Participante } & \multirow[t]{2}{*}{ Idade } & \multicolumn{2}{|c|}{ Motor } & \multicolumn{2}{|c|}{ Cognição } & \multicolumn{2}{|c|}{ Linguagem } & \multicolumn{2}{|c|}{ Psicossocial } & \multirow{2}{*}{$\begin{array}{c}\text { (Acertos) } \\
(\%)\end{array}$} \\
\hline & & Es & At & Es & An & Es & At & Es & At & \\
\hline 01 & $18 \mathrm{~m}$ & 9 & 7 & 4 & 4 & 4 & 1 & 3 & 3 & 75,6 \\
\hline 02 & $19 \mathrm{~m}$ & 7 & 6 & 3 & 3 & 5 & 3 & 5 & 5 & 86,4 \\
\hline 03 & $14 \mathrm{~m}$ & 11 & 9 & 7 & 7 & 6 & 4 & 8 & 8 & 87,1 \\
\hline 04 & $26 \mathrm{~m}$ & 7 & 6 & 3 & 2 & 5 & 4 & 5 & 5 & 83,0 \\
\hline 05 & $20 \mathrm{~m}$ & 7 & 7 & 4 & 3 & 4 & 2 & 6 & 6 & 81,2 \\
\hline 06 & $26 \mathrm{~m}$ & 7 & 6 & 3 & 3 & 5 & 2 & 5 & 4 & 49,4 \\
\hline 07 & $22 \mathrm{~m}$ & 9 & 9 & 4 & 4 & 4 & 3 & 3 & 2 & 85,4 \\
\hline 08 & $24 \mathrm{~m}$ & 7 & 5 & 3 & 3 & 5 & 4 & 5 & 5 & 87,8 \\
\hline 09 & $18 \mathrm{~m}$ & 7 & 5 & 3 & 3 & 5 & 5 & 5 & 5 & 92,8 \\
\hline 10 & $15 \mathrm{~m}$ & 11 & 1 & 7 & 1 & 6 & 0 & 8 & 2 & 12,0 \\
\hline 11 & $18 \mathrm{~m}$ & 7 & 4 & 4 & 4 & 4 & 4 & 6 & 6 & 89,2 \\
\hline 12 & $05 \mathrm{~m}$ & 11 & 10 & 7 & 7 & 6 & 6 & 8 & 8 & 97,7 \\
\hline 13 & $13 \mathrm{~m}$ & 7 & 6 & 4 & 4 & 4 & 2 & 6 & 4 & 75,6 \\
\hline 14 & $26 \mathrm{~m}$ & 7 & 5 & 3 & 3 & 5 & 5 & 5 & 5 & 92,8 \\
\hline 15 & $21 \mathrm{~m}$ & 9 & 8 & 4 & 4 & 5 & 5 & 4 & 4 & 97,2 \\
\hline 16 & $34 \mathrm{~m}$ & 11 & 7 & 7 & 2 & 6 & 3 & 8 & 5 & 51,1 \\
\hline 17 & $25 \mathrm{~m}$ & 13 & 11 & 3 & 3 & 2 & 0 & 2 & 1 & 58,6 \\
\hline 18 & $28 \mathrm{~m}$ & 9 & 8 & 4 & 4 & 4 & 1 & 3 & 3 & 78,4 \\
\hline 19 & $16 \mathrm{~m}$ & 13 & 11 & 3 & 3 & 2 & 2 & 2 & 2 & 96,1 \\
\hline 20 & $16 \mathrm{~m}$ & 9 & 9 & 4 & 4 & 4 & 2 & 3 & 3 & 87,5 \\
\hline 21 & $16 \mathrm{~m}$ & 9 & 9 & 4 & 4 & 4 & 2 & 3 & 3 & 87,5 \\
\hline 22 & $13 \mathrm{~m}$ & 7 & 3 & 3 & 2 & 5 & 5 & 5 & 4 & 72,3 \\
\hline 23 & $22 \mathrm{~m}$ & 13 & 8 & 3 & 2 & 2 & 0 & 2 & 2 & 57,0 \\
\hline 24 & $25 \mathrm{~m}$ & 7 & 6 & 3 & 1 & 5 & 1 & 5 & 4 & 54,7 \\
\hline 25 & $27 \mathrm{~m}$ & 11 & 9 & 7 & 7 & 6 & 5 & 8 & 5 & 81,9 \\
\hline 26 & $16 \mathrm{~m}$ & 9 & 9 & 4 & 4 & 4 & 2 & 3 & 3 & 87,5 \\
\hline 27 & $16 \mathrm{~m}$ & 9 & 9 & 4 & 4 & 4 & 2 & 3 & 3 & 87,5 \\
\hline 28 & $16 \mathrm{~m}$ & 8 & 6 & 2 & 1 & 1 & 1 & 2 & 2 & 81,2 \\
\hline 29 & $16 \mathrm{~m}$ & 13 & 9 & 3 & 2 & 2 & 2 & 2 & 2 & 83,9 \\
\hline 30 & $09 \mathrm{~m}$ & 7 & 2 & 3 & 3 & 5 & 5 & 5 & 5 & 82,1 \\
\hline
\end{tabular}


AMARAL, A. C. T.; TABAQUIM, M. L. M.; LAMÔNICA, D. A. C.

Tabela 3 - Representação numérica, por área, e média percentual dos desempenhos do GII, esperados (Es) e atingidos (At).

\begin{tabular}{|c|c|c|c|c|c|c|c|c|c|c|}
\hline Participante & Idade & \multicolumn{2}{|c|}{ Motor } & \multicolumn{2}{|c|}{ Cognição } & \multicolumn{2}{|c|}{ Linguagem } & \multicolumn{2}{|c|}{ Psicossocial } & Acertos (\%) \\
\hline & & Es & At & Es & At & Es & At & Es & At & \\
\hline 01 & $20 \mathrm{~m}$ & 1 & 1 & 1 & 1 & 4 & 3 & 1 & 1 & 93,7 \\
\hline 02 & $18 \mathrm{~m}$ & 8 & 6 & 2 & 2 & 1 & 1 & 2 & 2 & 93,7 \\
\hline 03 & $16 \mathrm{~m}$ & 8 & 6 & 2 & 2 & 1 & 1 & 2 & 2 & 93,7 \\
\hline 04 & $27 \mathrm{~m}$ & 1 & 1 & 1 & 1 & 4 & 4 & 1 & 1 & 100 \\
\hline 05 & $20 \mathrm{~m}$ & 1 & 1 & 1 & 1 & 4 & 4 & 1 & 1 & 100 \\
\hline 06 & $28 \mathrm{~m}$ & 1 & 1 & 1 & 1 & 4 & 4 & 1 & 1 & 100 \\
\hline 07 & $24 \mathrm{~m}$ & 1 & 1 & 1 & 1 & 4 & 4 & 1 & 1 & 100 \\
\hline 08 & $24 \mathrm{~m}$ & 1 & 1 & 1 & 1 & 4 & 4 & 1 & 1 & 100 \\
\hline 09 & $18 \mathrm{~m}$ & 8 & 6 & 2 & 2 & 1 & 1 & 2 & 2 & 93,7 \\
\hline 10 & $15 \mathrm{~m}$ & 9 & 9 & 4 & 4 & 4 & 4 & 3 & 3 & 100 \\
\hline 11 & $18 \mathrm{~m}$ & 8 & 6 & 2 & 2 & 1 & 1 & 2 & 2 & 93,7 \\
\hline 12 & $05 \mathrm{~m}$ & 7 & 7 & 3 & 3 & 5 & 5 & 5 & 5 & 100 \\
\hline 13 & $13 \mathrm{~m}$ & 7 & 7 & 4 & 4 & 4 & 4 & 6 & 6 & 100 \\
\hline 14 & $26 \mathrm{~m}$ & 1 & 1 & 1 & 1 & 4 & 4 & 1 & 1 & 100 \\
\hline 15 & $22 \mathrm{~m}$ & 1 & 1 & 1 & 1 & 4 & 4 & 1 & 1 & 100 \\
\hline 16 & $34 \mathrm{~m}$ & 1 & 1 & 1 & 1 & 4 & 4 & 1 & 1 & 100 \\
\hline 17 & $24 \mathrm{~m}$ & 1 & 1 & 1 & 1 & 4 & 4 & 1 & 1 & 100 \\
\hline 18 & $27 \mathrm{~m}$ & 1 & 1 & 1 & 1 & 4 & 4 & 1 & 1 & 100 \\
\hline 19 & $16 \mathrm{~m}$ & 9 & 9 & 4 & 4 & 4 & 4 & 3 & 3 & 100 \\
\hline 20 & $16 \mathrm{~m}$ & 9 & 8 & 4 & 4 & 4 & 4 & 3 & 3 & 97,2 \\
\hline 21 & $14 \mathrm{~m}$ & 9 & 8 & 4 & 4 & 4 & 4 & 3 & 3 & 97,2 \\
\hline 22 & $13 \mathrm{~m}$ & 9 & 9 & 4 & 4 & 4 & 4 & 3 & 3 & 100 \\
\hline 23 & $22 \mathrm{~m}$ & 1 & 1 & 1 & 1 & 4 & 4 & 1 & 1 & 100 \\
\hline 24 & $25 \mathrm{~m}$ & 1 & 1 & 1 & 1 & 4 & 4 & 1 & 1 & 100 \\
\hline 25 & $27 \mathrm{~m}$ & 9 & 9 & 4 & 4 & 4 & 4 & 3 & 3 & 100 \\
\hline 26 & $16 \mathrm{~m}$ & 8 & 8 & 2 & 2 & 1 & 1 & 2 & 2 & 100 \\
\hline 27 & $16 \mathrm{~m}$ & 9 & 9 & 4 & 4 & 4 & 4 & 3 & 3 & 100 \\
\hline 28 & $18 \mathrm{~m}$ & 1 & 1 & 1 & 1 & 4 & 4 & 1 & 1 & 100 \\
\hline 29 & $16 \mathrm{~m}$ & 9 & 9 & 4 & 4 & 4 & 4 & 3 & 3 & 100 \\
\hline 30 & $09 \mathrm{~m}$ & 7 & 7 & 3 & 3 & 5 & 5 & 5 & 5 & 100 \\
\hline
\end{tabular}

Quanto ao desempenho geral, as Figuras de 1 a 5 demonstram os escores percentuais obtidos no grupo experimental (GI) correlacionado ao grupo controle (GII), quanto ao desempenho nas áreas avaliadas. A Figura 1 apresenta a média global dos resultados do total dos acertos, considerando todas as áreas avaliadas.

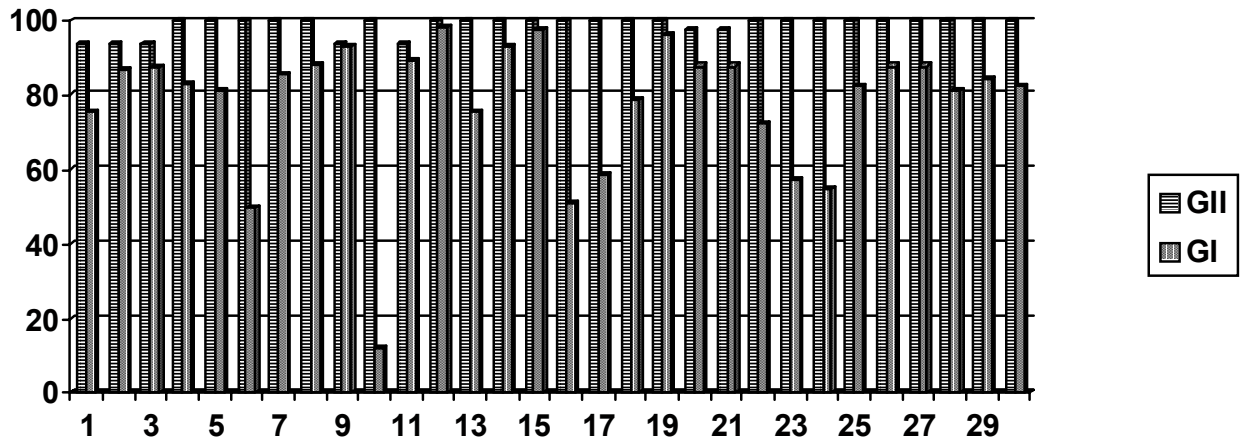

Figura 1 - Comparação dos desempenhos dos GI e GII, quanto à média global dos resultados. 
A Figura 2 refere-se aos resultados percentuais dos grupos controle (GII) e experimental (GI) quanto ao desempenho na área motora

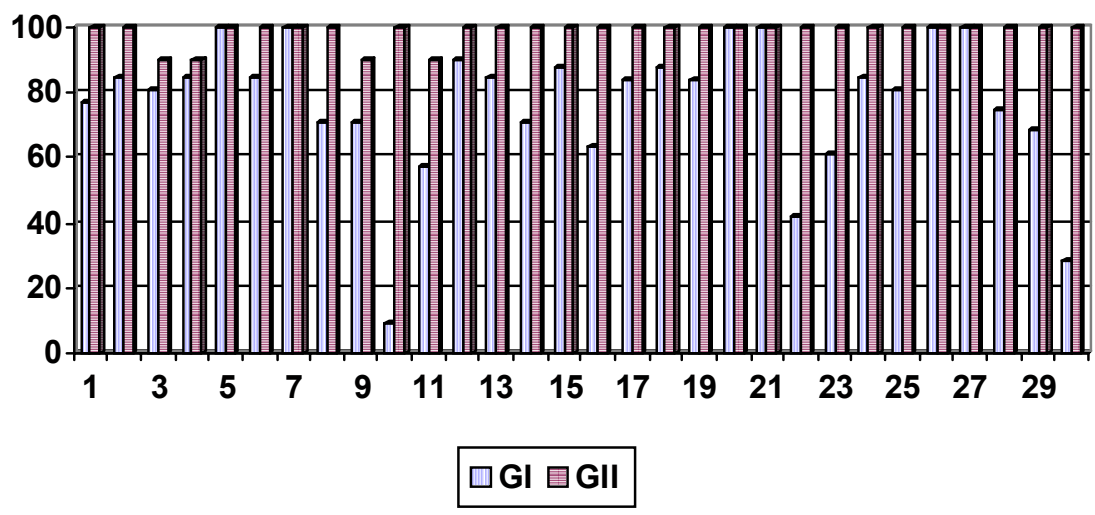

Figura 2 - Representação do desempenho motor do GI e GII.

A Figura 3 refere-se aos resultados percentuais dos grupos controle (GII) e experimental (GI) quanto ao desempenho na área cognitiva

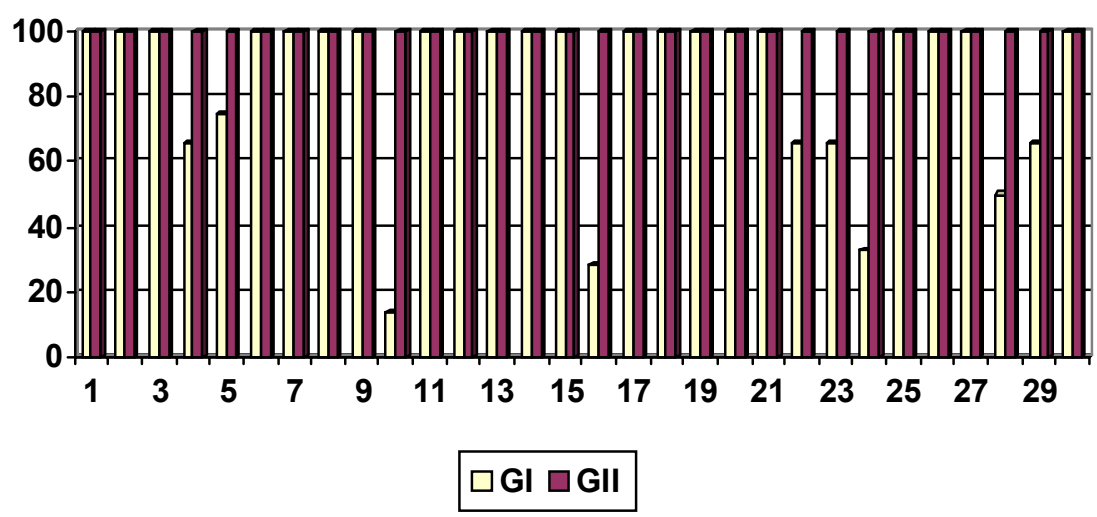

Figura 3 - Representação dos desempenhos cognitivos dos GI e GII.

A Figura 4 refere-se aos resultados percentuais dos grupos controle (GII) e experimental (GI) quanto ao desempenho na área de linguagem. 


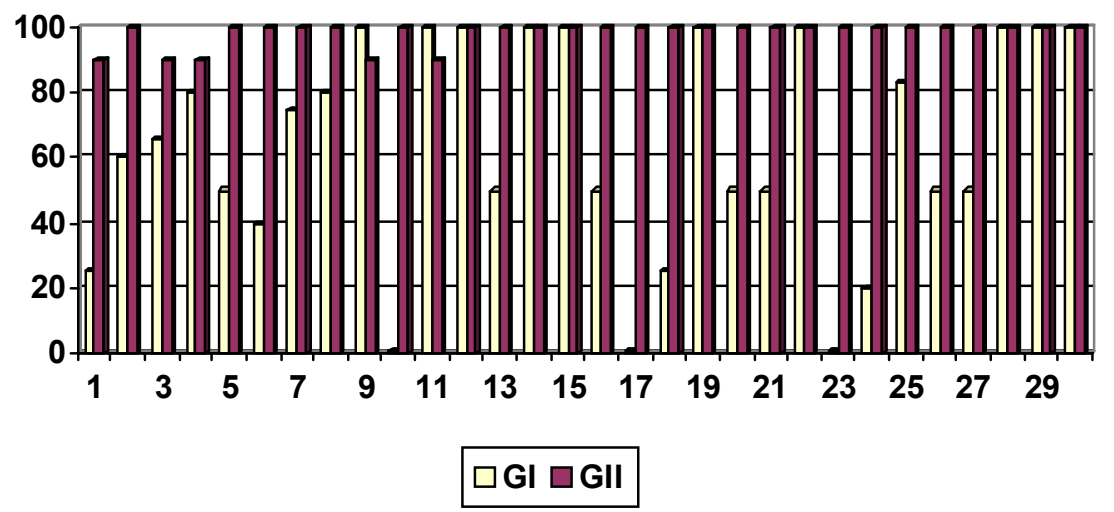

Figura 4 - Representação dos desempenhos de linguagem dos GI e GII.

A Figura 5 refere-se aos resultados percentuais dos grupos controle (GII) e experimental (GI) quanto ao desempenho na área psicossocial.

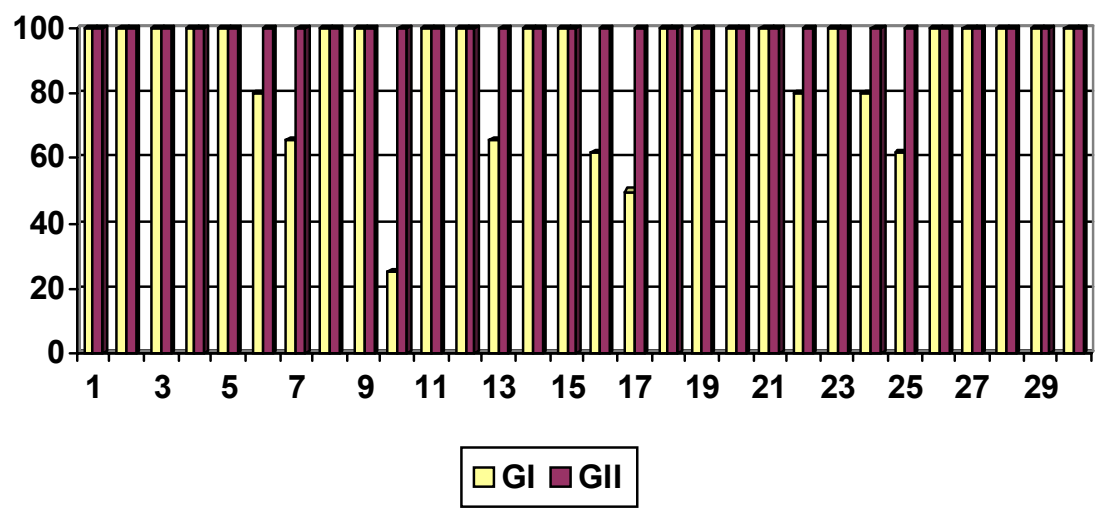

Figura 5 - Representação dos desempenhos psicossociais dos GI e GII.

A análise estatística por meio dos testes Kruskal Wallis e teste Miller indicou que na comparação entre os grupos GI e GII, quando comparado o desempenho de cada área, as áreas motora $(p=0.002695)$, cognitiva $(p=0,016421)$ de linguagem $(p=0.00038)$ apresentaram diferenças estatisticamente significantes. Somente a área psicossocial $(\mathrm{p}=0,221943)$ a comparação entre os grupos não confirmou esta diferença.Verificando a correlação entre as áreas a área de maior prejuízo é a de linguagem, seguida da motora, cognitiva e psicossocial, esta última aquela com menor prejuízo, considerando os escores analisados. 


\section{Discussão}

Considerando que quando há atraso do desenvolvimento neuropsicomotor, o sistema nervoso encontra-se imaturo e em plena fase de desenvolvimento (DIAMENT, CYPEL, 1996), as manifestações que caracterizam o atraso desenvolvimental, por imaturidade, podem ser alteradas e as dificuldades iniciais superadas, justificando que o diagnóstico neurológico não se encontre totalmente estabelecido. Isto ocorreu neste estudo e por esta razão optou-se pela não utilização de rótulos diagnósticos. Cabe ressaltar que com o tratamento e estimulações adequadas ocorrendo precocemente, crianças imaturas podem evoluir e apresentar, após este processo, desenvolvimento adequado, considerando que a estimulação ativa pode proporcionar organização da plasticidade neuronal (HAIG, 1995; LEVI, 2003).

Nesta amostra constatou-se que $74 \%$ apresentaram prematuridade e baixo peso e $60 \%$ histórico de hipoxemia. A associação da prematuridade como fator de risco já é bem conhecida (SUSSMAN, 1996; LEVI, 2003). No entanto, é comum aceitar que o sofrimento perinatal é responsável pela maioria dos casos intercorrentes, sem considerar que este possa decorrer de uma condição pré-natal já existente e predisponente aos efeitos de um parto traumático (TABAQUIM, 2002).

$\mathrm{Na}$ representação do desempenho motor de ambos os grupos, apresentados na Figura 2, observou-se que os resultados de GI com relação ao GII, encontram-se inferiores à performance esperada para a faixa etária. $\mathrm{O}$ atraso do desenvolvimento motor é evidente no GI, trazendo dificuldade para autonomia e exploração destas crianças no seu ambiente. $\mathrm{O}$ atraso neuropsicomotor pode proporcionar atraso em outras áreas, pois sua presença limita a criança na ação de seu ambiente, no refinamento dos atos motores, na recorrência das ações, na experimentação e atividades interativas com objetos e pessoas, ou seja no ambiente no qual o processo de aprendizagem se desenvolve (BACKLIN, 1992; LAMÔNICA, 2000; MILLER E CLARK, 2002). O estudo de Pheifer (1997) chamou a atenção para este aspecto, seus achados indicaram que as crianças que apresentaram problemas motores tiveram desempenhos inferiores em habilidades perceptivas, pois, muitas destas são construídas na interação motora.

Quanto à cognição (Figura 3) 70\% do GI apresentou os aspectos cognitivos preservados. Cabe ressaltar que a literatura apresenta que nem todos os indivíduos que apresentam alterações motoras apresentam alterações cognitivas, mesmo com a influência negativa que o transtorno motor pode causar no desenvolvimento dos processos cognitivos (GERBER, 1996; SORO, BRASIL,1997; MILLER, CLARK, 2002; BERGER, 2003). Considerando que o desenvolvimento cognitivo emerge das interações de vários sistemas somáticos, ou seja, integrações neurofuncionais entre o corpo e o cérebro, pode ser afetado por pressões patológicas inerentes às condições intercorrentes. As respostas adaptativas, observadas neste estudo, demonstraram maiores prejuízos cognitivos nos níveis mais complexos e simbólicos da motricidade, e, ganhos nos mais simples e elementares. 
Quanto à linguagem (Figura 4) constatou-se que os resultados obtidos envolvendo esta área, representaram os menores escores, enquanto área avaliada. Uma das condições que podem promover alterações de linguagem é a limitação motora decorrente da dificuldade de exploração e interação com o meio (LAMÔNICA, 2004). É sabido que a criança nos dois primeiros anos de vida, na vivência do período sensório-motor, necessita agir no seu ambiente, manipular objetos, interagir independentemente, para que favoreça a formação de representações sociais que decorrem destas condições. A criança quando em condições de limitação motora, torna-se privada dos meios que possam auxiliá-la no desenvolvimento de habilidades motoras, cognitivas, sociais e da comunicação, promovendo a organização da linguagem em seus aspectos semânticos, pragmáticos e sintáticos (BACKIN, 1992; PFEIFER, 1997; MARUJO, 1998; LAMÔNICA, 2004).

A área da linguagem mostrou-se destacada quanto a prejuízos. Este aspecto pode estar relacionado à falta de experiências de exploração da criança no meio e como a participação ativa é diminuída, causa um descompasso na velocidade e compreensão dos aspectos lingüísticos.

De todas as áreas avaliadas, a psicossocial demonstrou ser a de maior domínio para todos os participantes do estudo, tanto para o grupo experimental quanto o controle. No grupo GII, os participantes obtiveram 100\% de acerto nas provas e no GI 70\%. Esta foi a única categoria que, o cálculo estatístico, na comparação entre os grupos os resultados não apresentaram ser estatisticamente significantes. Infere-se que esta habilidade, relacionada com as atividades de interação interpessoal e familiar, decorreu da valorização do meio à essas habilidades, na estimulação e resposta da criança como fonte inclusive de gratificação.

Portanto, identificar precocemente a criança de risco e suas necessidades por meio de procedimentos avaliativos específicos, permite não só a compreensão do fenômeno, como também evita maiores prejuízos no seu desenvolvimento.

\section{Conclusões}

O presente estudo, ao investigar as condições de crianças nos dois primeiros anos de vida, que tiveram história clínica de risco concluir que na comparação entre grupos, (GI e GII) o grupo GI apresentou diferença estatisticamente significante nas áreas motora, de linguagem e cognitiva. Na análise da comparação entre áreas, as áreas de maior prejuízo foram a área da linguagem e motora, seguido da cognitiva. A área psicossocial foi a menos comprometida.

Este estudo permitiu concluir que, na avaliação das habilidades em crianças de risco, deve-se atentar para o desenvolvimento motor, da linguagem e cognitivo assim como, considerar a importância dos aspectos preventivos e interventivos, na otimização dos recursos estruturais e funcionais da criança em desenvolvimento. 


\section{REFERÊNCIAS}

ATKINSON, R.L.; ATKINSON, R.C.; SMITH, E.E.; BEM, D.J.; NOLEN-HOEKSEMA, S. Introdução à neuropsicologia. Porto Alegre: Artmed, 2002.

BACKIN, J.S. Your child's development. In: GERALIS, E. Children with cerebral palsy: A parent's guide. Woodbine House, 1992, p.175-208.

BERGER, K.S. O desenvolvimento da pessoa da infância à adolescência. Rio de Janeiro: LTC editora, 2003.

CIPRIANI, P.; BRUNI, G. Relations between sensoriomotor attainment and development of language comprehension in retard child. Italian J. Neurol Sci. P.133-141, 1986.

COHEN, H. Neurociências para fisioterapeutas - incluindo correlações clínicas. São Paulo: Manole, 2001.

GERBER, A. Problemas de aprendizagem relacionados à linguagem - sua natureza e tratamento. Porto Alegre: Artes Médicas, 1996.

HAIG, D. Prenatal power plays: natural history. New Jersey: Ablex, 1995.

LAMÔNICA, D. A. C. Diagnóstico fonoaudiológico: reconhecimento semântico e reconhecimento de frases acusticamente distorcidas (PSI) em paralíticos cerebrais. 2000. 258f. Tese (Doutorado) - Programa de Distúrbios da Comunicação Humana - Universidade Federal de São Paulo, São Paulo.

LAMÔNICA, D. A. C. Linguagem na paralisia cerebral In: FERREIRA, L. P.;BEFI-LOPES, D. M.; LIMONGI, S.C.O. Tratado de Fonoaudiologia. Roca, São Paulo, 2004. p.967-976.

LEVY, J. A; OLIVEIRA, A.S.B. Reabilitação em doenças neurológicas: guia terapêutico pratico. São Paulo: Atheneu, 2003.

LYONS, P.; RITTNER, B. The construction of the crack babies phenomenon as a social problem. American Journal of Orthopsychiatry. v 68, 313-320, 1998.

MARUJO, V.L.M.B. Fonoaudiologia em paralisia cerebral. In: SOUZA, A.M.C.; FERRARETTO, I. Paralisia cerebral: aspectos práticos. São Paulo: Memnon, 1998.

MILLER, G.; CLARK, G.D. Paralisias cerebrais: causas, conseqüências e condutas. São Paulo: Manole, 2002.

PIOVESANA, A.M.S.G. Hemiparetic cerebral palsy: etiological risk factors and neuroimaging. Arquivos de Neuropsiquiatr., v. 59, n 1, p. 29-34, 2001.

PHEIFER, L.I. Comprometimento motor e aquisição de habilidades cognitivas em crianças portadoras de paralisia cerebral. Temas em Desenvolvimento, v 6, n 31, p. 4-13, 1997.

PIOVESANA, A.M.S.G. Paralisia cerebral: contribuições do estudo por imagem. In: SOUZA, A.M.C.; FERRARETTO, I. Paralisia Cerebral: aspectos práticos. São Paulo: Memnon, 1998.

SAES, S.O. Avaliação do aparecimento da habilidade de localização sonora em crianças com e sem alterações timpanométricas. 2003. Tese (Doutorado) - Universidade Estadual Paulista "Júlio de Mesquita", Botucatu.

SORO, C.E.; BRASIL, C. Desavrollo de la comunicación y el lenguajeen ninõ con descapacidad motora y plurideficiença. In: DEL RIO, M.J. Interaccion y desavrollo del lenquajr en personas con necessidades especiales. 1997. Conclusões p.87 - 112. 
AMARAL, A. C. T.; TABAQUIM, M. L. M.; LAMÔNICA, D. A. C.

SUSSMAN, S.I.T.; GLYNN, S.M. STACY, A.W. What does "high risk" mean? a psyc info scan of the literature. Behavior Thepary, v. 27, p. 53-65, 1996.

TABAQUIM, M.L.M. Avaliação neuropsicológica: estudo comparativo de crianças com paralisia cerebral hemiparética e distúrbios de aprendizagem. 2002. Tese (Doutorado) Faculdade de Ciências Médicas - Unicamp, Campinas.

Recebido em 26/02/2005

Reformulado em 26/02/2005

Aceito em 26/07/20505 